\title{
15
}

\section{Identifying Research Strategies and Methodological Priorities for the Study of Demanding Energy}

\section{Allison Hui, Rosie Day, and Gordon Walker}

As the introduction established, there is considerable variation in accounts of energy demand. Starting from different places establishes different priorities and prompts different types of insights. This final chapter revisits the diverse chapters of the book with an explicit focus upon research strategies and how they relate to a shared interest in demanding energy. It recognises that explicitly discussing research tools and techniques can go some way to help stimulate what Mills (1970) might call the methodological imagination, and perhaps along the way make researching demanding energy less demanding for others. Explicitly attending to the research strategies used by the authors in this book is therefore part of developing a research agenda that places processes of demanding energy as its central concern.

The primary question underlying all of the studies discussed in this book is: what is energy for? This question builds upon the assumption

A. Hui $(\bowtie) \bullet$ G. Walker

Lancaster University, Lancaster, UK

R. Day

University of Birmingham, Birmingham, UK

(C) The Author(s) 2018

A. Hui et al. (eds.), Demanding Energy, DOI 10.1007/978-3-319-61991-0_15 
that energy is not used for its own sake, but in the course of performing a range of social practices that have distinctive spatio-temporal characteristics and dynamics of change. The social world, however, is vast, and given both the pervasiveness of energy and the on-going spread of energy infrastructures across many regions of the world, studying what energy is for could ostensibly lead to examining any part of social life. It is instructive therefore to reflect upon how the authors in this book have gone about their research, through composing research questions to address carefully defined concerns, delimiting suitable cases and selecting appropriate samples and methods.

The foregrounding of research strategies is deliberate. Whilst authors used a range of methods - interviews, ethnography, time use surveys, the analysis of secondary and visual data-these are not themselves particularly distinctive in relation to social scientific research in general, or energy research in particular. More innovative and consequential are the types of questions and aims that authors set out to investigate. These, in conjunction with the approaches to sampling and settings that follow from them, establish research designs probing aspects of demanding energy that have been understudied. This chapter thus begins by considering research questions and the priorities embedded in them. It then moves on to consider issues related to the selection of cases and samples, before concluding with some more general observations.

\subsection{Methodological Priorities and Their Research Design Implications}

While methodology and methods are often conflated in discussions of research, the former can be seen to involve not only techniques but also specific claims about the type of knowledge that is produced using particular techniques. Interviews or participant observation are therefore methods for gathering data, and thematic analysis through coding is one technique of working with that data. Thinking about methodology requires a broader view - a consideration of how the topic at hand is being approached and investigated, and what kind of knowledge is generated as a result. It is therefore about, for example, 
how research questions are phrased, which units of study are chosen and what research designs are able to investigate.

In the introduction to this book we argued that starting from a concern for what energy is for generates different understandings of how energy demand is constituted, patterned and changing. That is, it generates different knowledge about demanding energy. We are now in a position to reflect upon that argument in terms of methodology_-if studying demanding energy generates new types of knowledge then this is in part due to particular methodological priorities. Another way of conveying this is that pursuing the question 'what is energy for?' involves a methodological approach that prioritises specific things during the framing of research questions, the defining of units of study and the formulation of research designs. Together, these priorities facilitate the production of a different type of knowledge about demanding energy.

Three methodological priorities that are embedded in this collection are outlined below. The specific formulation is ours-authors may not have engaged with these priorities explicitly within their own projects, but we argue that their work attends to them nonetheless. Nor is this list intended to be exhaustive — other shared priorities may also be found. The three methodological priorities outlined here, however, can be seen as points of intersection and agreement between authors, and provide a means of considering how the insights from one case might be considered in relation to other cases. They can also be read as prompts for future research-priorities that other researchers might embed into their own research questions and designs, and that policymakers might consider when looking for evidence to support particular interventions.

Priority one: it is crucial to pose questions that focus upon social dynamics rather than upon energy itself. Examples within this collection include: How is eating changing over time? What practices make up temporary events like music festivals? How is work within different departments in a hospital interconnected? How does leisure travel fit into the lives of diverse older people? Such questions are of significant relevance for discussions of past, present or projected energy demand, but they seek to first understand practices on their own terms. This is important because it acknowledges that everyday practices are organised around a wide variety of aims and goals (see discussion of timespace and human activity in 
Schatzki 2010), aims that are often completely unrelated to energy for those engaged in them. One does not stay in a hotel in order to support energy demand management efforts - one stays at a hotel because of being away from home and needing somewhere to sleep overnight. Nevertheless, staying in a hotel has an impact on energy demand, its spatiality and temporality, and in aggregate, this is a significant effect. Collecting more detailed data on what is happening in the social world is thus crucial, and can reveal complexities and variations in processes of demanding energy that have been overlooked in the generation of existing projections, policies and paths for future change. Rather than assuming that cooking and car driving are performed similarly across a population, have universal meanings, or might be changed using similar strategies (e.g. campaigns asking people to make different choices), approaching them as topics of study brings into empirical question the extent to which they vary and the complex interactions that affect their transformation. Directing attention away from energy thus opens up opportunities for rich understandings of how social life is organised, and as a result paves the way for more sophisticated understandings of how demanding energy is a part of it.

Priority two: it is important to reflect upon how particular units of study facilitate the examination of different types of interconnections - for example within and between practices, technologies or organisations. Whilst the study of what energy is for can draw upon theories of practice, it is not synonymous with the study of practices (praktik) as understood within this literature (e.g. Reckwitz 2002). As we noted in the introduction, some of the authors in this collection do not draw in detail upon theories of practice, but rather build upon a broader social scientific literature interested in what people do. Whilst the concepts developed within theories of practice are particularly useful for developing understandings of what energy is for, they are not the only relevant ones. In addition to this general point, however, is a more specific one. To date, many studies applying theories of practice to the study of sustainability, climate change and related energy issues have been concerned with defining specific practices made up of constituent elements-for example using Shove et al.'s three-element model (2012). This is not, however, a necessary part of research designs. Starting out by defining practices of interest is only 
one of many research strategies available to those working more closely with theories of practice.

As our authors demonstrate, we can learn just as much about what energy is for by looking at how technologies are connected with each other and integrated into people's activities. Whilst at first glance it may seem this replicates the interest in technologies found in other energy literature, there is a key difference from much of this work. Rather than taking particular technologies or infrastructures as a primary focus, as found in user adoption studies or discussions of energy efficiency, authors interrogate how their interconnection with other technologies affects the process of demanding energy. For example, Wiig is interested not simply in mobile phones or tablets, but in how these are connected to infrastructures that facilitate their charging and mobile connectivity. He also investigates how infrastructures are themselves interwoven-with a provider of rail transport becoming increasingly involved in the provision of digital connectivity. A further example is Sahakian, whose study of household practices positions appliances as points of intersection between affluent householders, hired domestic helpers and architects or designers who each affect, in different ways, 'normal' practices. Curtis et al.'s discussion of demand management in hotels similarly considers not only the technologies and automated processes that make demand management schemes possible, but also how these materials connect to a series of activities — of which some are automated whilst others require the input of a range of employees in multiple sites. The use of one technology is therefore always connected, theoretically and empirically, to the use of other technologies as part of a wider system of practices that demands energy. As these chapters demonstrate, rather than asking questions about how technologies can be made more energy efficient or how more sustainable technologies can be more widely adopted, studying demanding energy involves asking different questions: How are norms around the use of technologies developed and reproduced? How do groups of people develop, maintain and use technologies in conjunction with particular infrastructures? How do multiple infrastructures interrelate materially and socially? The implication of these questions is that technologies have no unique importance within processes of demanding energy-rather, they are part of both infrastructural and normative systems that need to 
be empirically studied. Research questions, empirical data and analyses must therefore seek to query and retain at least some these connections.

What energy is for can also be investigated by focusing empirical work on organisational units and then investigating some of the many practices that occur within them. This approach provides not only an opportunity to consider how multiple practices are interconnected, but also to push against the segmentation of practices into discrete domains-as with the isolation of transport research from energy research. Many things take place in organisations, and starting from these units can prompt different kinds of insights about what energy is for and how demanding energy takes place. Blue's research focuses upon the work done within hospital settings, taking as a starting point the idea that interconnections between different departments and divisions are extremely consequential for understanding how patient care is delivered, and how energy is demanded as a consequence. Similarly, Jones et al. demonstrate in their study of construction and engineering consulting firms that the procuring and arranging of work within a firm has significant implications for the arrangement of 'necessary' travel. Taking a slightly different focus, Allen shows how 'events' can be seen as composed of a variety of practices. By thinking through live music events in rural and urban contexts, he raises important questions about how processes of demanding energy are situated in relation to diverse infrastructures. We might even think about the household as a kind of organisational unit, within which the diverse practices addressed by Greene and by Douzou and Beillan take place. In these studies, focusing on organisational units is thus not about providing analyses of those units per se. Instead, it is about thinking through other types of questions: How do dependencies and connections between departments affect the flow of work (and of energy)? How does the changing of one set of organisational practices affect other practices (and how they demand energy)? What are the implications for energy demand when organisations and events take on new practices that were not previously their concern (as with 'glamping' at music festivals)? How do the practices of household units bring into question the boundaries of home spaces? These questions recognise that the process of demanding energy is shaped by a range of organisations that structure work, generate expectations for co-presence and travel, 
coordinate infrastructures and inhabit varied spaces, among other things. Research questions, empirical data and analyses would therefore helpfully address and acknowledge these links.

Whilst different research questions are generated by approaching the study of what energy is for in each of these ways - from the definition of key practices of interest, from the systemic connections of particular types of technologies and from the designation of particular organisational units of interest-it is also possible to see how these approaches might also provide complementary insights. For example, Blue's discussion of CT scanner technology might have also been taken as the starting point for a more technology-focused investigation akin to that found in Wiig's chapter. Conversely, it would be possible to undertake a more institutionally focused study of Amtrak, the train company discussed in Wiig's chapter, and how the provision of digital connectivity, in addition to mobility, became part of its mandate. In this way the approaches used by different authors in the book offer possibilities for further investigating the range of cases presented here, as well as many others.

Priority three: whether research designs privilege practices, technologies or organisational units, it is methodologically important that they incorporate spatial and temporal dynamics. This is because what energy is for changes. Demanding energy is constituted by processes situated in specific locations marked by particular material and temporal relationships. The proximity of co-workers, the temporariness of infrastructures, the fluctuations of seasonal holidays, the rhythms of days and life cycles all affect how practices are performed and how energy is demanded. It is therefore important to build in opportunities for these dynamics to be recorded and analysed through research designs that probe variations and encourage comparisons across space, over time and in relation to practice-specific transformations. Whilst all of the chapters speak to these dynamics in some manner, those by Greene, Durand-Daubin and Anderson, and Jones et al. are particularly interesting for how they engage with trajectories of change in relation to different units- people, practices and institutions, respectively. Their work highlights how different trajectories of change are simultaneously shaping processes of demanding energymeaning as a result that attempts to shape or steer future change will also encounter these different dynamics. Whilst this is a complex process, 
collecting more data that facilitates a consideration of units in relation to multiple, evolving spatial and temporal dynamics will provide a valuable basis of evidence. Research designs can therefore usefully consider questions such as: How do particular spatial relations matter to what energy is for? What temporal relations and variations can be observed? What exactly is changing over time and how might it be documented?

These three methodological priorities are embedded, in different ways, into the more specific questions that authors address in their empirical research. Whilst broadly cast, we argue that together they present a distinct approach that distinguishes the study of demanding energy from existing studies of energy demand. As such, they may be usefully taken up and developed by others interested in exploring new approaches to the study of energy demand.

Whilst these priorities end up being embedded into core research questions and the specifics of research designs, there are other techniques and tools that are also important within research strategies. The next section addresses one set—related to cases and sampling - in more detail, as this aspect of research design is very important for creating links between energy and social practices.

\subsection{Approaching Cases and Sampling}

Whilst asking what energy is for can prompt research questions that direct attention away from energy itself, it is of course important that it is not forgotten. We suggest that one of the aspects of research design where existing knowledge about energy demand is most central is in the specification and sampling of cases. As noted earlier, nearly any social process or practice could be studied and then connected, in some way, to energy. Attending to some cases and not others can therefore be justified on the basis of their importance for demanding energy.

Working through case studies in the first place could, in some energy research communities, be seen as a weakness. Positivistic approaches to research remain prevalent in non-social scientific disciplines, and therefore the acceptance of case study research that exists within the social sciences can be brought into question within interdisciplinary discussions 
of energy demand. However, in his powerful defence of case studies, Flyvbjerg notes that their utility is not limited to generating hypothesis or providing pilot insights, despite this widespread impression (2001: 77). Rather, he argues, careful sampling can make it possible to theorise on the basis of single case studies. What he calls information-oriented selection of samples and cases, based on expectations about the type of data they will provide, can be used to carefully address gaps in knowledge and provide important insights. In particular, he names three types of information-oriented selection that are particularly valuable when studying what energy is for:

1. Extreme/deviant cases: To obtain information on unusual cases, which can be especially problematic or especially good in a more closely defined sense.

2. Maximum variation cases: To obtain information about the significance of various circumstances for case process and outcome[...]

3. Critical cases: To achieve information which permits logical deduction of the type, if this is (not) valid for this case, then it applies to all (no) cases. (Flyvbjerg 2001: 79)

Each of these types of cases provides a means of linking insights to other cases and contexts not on the basis of representativeness, but on the basis of other exemplary, relational or logical ties.

Whilst case-based studies of demanding energy may therefore depart from some of the selection and sampling procedures established in other niches of energy research, the insights and assumptions from existing studies can prove central to case selection. The first example of this can be seen in the extreme case of Sahakian's Geneva expats. The experiences of this group of participants are not likely to be familiar to or common amongst the wider population in Switzerland, or other developed nations. But in energy terms they are interesting precisely because they, and the houses they inhabit, are out of the ordinary. Given the increase over time in the number of appliances built into houses and used by households, looking at a community where multiple appliances facilitate household practices can be argued to provide important insights on a case that might be deemed particularly problematic in energy terms. Rather than just 
understanding this group, it offers the potential to consider how levels of 'normal' energy use are established and maintained, when these norms are excessive in comparison to many other groups and contexts. It is not necessary that such an extreme case of energy use should be treated as a possible future norm for everyone. Indeed, this kind of projection can be problematic because of how it ignores the diverse trajectories of change at work. Nonetheless, investigating an extreme case can make it easier to see some of the relations between everyday practices, infrastructures, technologies and social norms that are easily overlooked when studying more familiar contexts.

Whilst none of the chapters in this collection focus centrally upon maximum variation cases, we can see that this approach to selection is apparent in some of their samples. Day et al., for example, segment their participants into three groups of older people, in order to more carefully consider the variations within older populations and how this affects travel demand. As their data shows, there are considerable differences in the embodied experiences and care responsibilities of different groups of older people, and these are consequential for imagining future patterns of travel demand. Similarly, Burkinshaw's project involved the selection of participants in different professions (architects, academics, graphic designers, accountants, solicitors and university support staff) that were expected to have contrasting access to flexible working. Whilst in the end he found no evidence of differential flexible working policies amongst these groups, this negative result was itself useful in determining the meaningful types of variation for discussions of working and commuting. The interest of many authors in spatial and temporal comparisons might also be seen as an important version of this selection strategy. In some chapters — such as Durand-Daubin and Anderson's work-the extent of this variation is constrained to some extent by existing datasets. Allen's chapter, however, demonstrates that pointing to variation-through reference to a wide range of different types of events - can be conceptually useful even where it is not tied to new empirical data. Engaging with varied cases or samples can thus be positioned to contribute a range of important insights about demanding energy.

Finally, building upon established knowledge about energy demand is most crucial for the selection of critical cases, which can be understood as 
framed in relation to assumptions. If a prevailing assumption is not true in a case where it might be most expected, then it makes sense to conclude that it is likely not true anywhere. Understanding existing assumptions about energy demand, and what energy is for, is thus important for those seeking to study critical cases. Several chapters of this book connect with this approach. Mullen and Marsden, Burkinshaw and Day et al. all frame their contributions in relation to existing arguments or assumptions about travel and how practices of travel are changing. These projections and suggestions about future change have been formulated in other energy research and policy contexts, but without detailed attention to whether they appropriately represent social dynamics. Therefore our authors, having chosen cases that put these assumptions to the test, are able to provide new insights that effectively dismantle them. Along the way, they highlight additional dynamics that need to be more carefully considered when developing future policies: the effect insecure working conditions have upon travel patterns; the interlinking of commuting with other types of travel and family practices; the importance of ageing bodies when considering leisure travel. In a different way, Douzou and Beillan, and Psarikidou, also position their arguments in relation to existing assumptions related to energy demand. Yet the assumptions they take on are not about how demand will or could change, but rather about its spatial and temporal characteristics. As a result, they are able to highlight how spatio-temporal meanings are quite complex. This complexity sits in contrast to (and indeed challenges) research using rational choice models that emphasise only the 'correct' attitudes and understandings that prompt choices aligned with sustainability or energy management objectives. Selecting critical cases thus provides a means of questioning and challenging existing assumptions about energy demand, whilst also generating data focused more upon demanding energy.

As these examples illustrate, careful consideration of case selection can ensure that even small studies or single cases can provide insights of relevance to broader considerations of demanding energy. In line with the second research priority above, cases may be of practices, of specific sets of people, perhaps in specific places or circumstances, of sets of technologies, or of organisations or settings. In conjunction with carefully formulated research questions, cases can be used to test or enter into dialogue 
with existing energy demand research, providing rich data about the social practices, spatio-temporal relations and transformations that affect what energy is for.

\subsection{Conclusion}

In researching processes of demanding energy, the authors in this collection have posed questions that foreground different concerns and enact different boundaries than other studies. For those working within particular empirical contexts or addressing energy demand in particular industries and policy realms, this will be apparent in terms of what is and isn't discussed and the specific insights provided by each chapter. This final chapter, however, has also demonstrated that these chapters have been marked by a distinct research approach. Starting from the question of what energy is for leads to different types of research questions that interrogate varied, interconnected units_-practices, technologies, institutions and social groups-and how they are positioned in relation to space, time and change. Most centrally, this research strategy challenges the idea that energy research must place energy at the centre of all of its research questions; see in this respect a recent debate about whether or not social science research on energy should be expected to engage explicitly with energy units and measures in order to achieve policy relevance (Cooper 2017; Castree and Watt 2017). Authors have instead demonstrated that asking relevant questions about social life, in relation to carefully selected cases, can provide valuable understandings of how demanding energy is constituted, patterned and changing.

Opening up a discussion about research strategies has highlighted that researching what energy is for depends less upon particular methods than upon ensuring that the generation of research questions and selection of cases and samples is theoretically engaged and closely informed by an interest in social practices on their own terms. Starting from this point creates opportunities to develop more sophisticated representations of how demanding energy works - ones that for example can narratively connect the activities of those using mobile devices with the activities of those developing digital infrastructures to support them. On one hand this collection has exemplified how specific projects, with carefully positioned 
research designs, can contribute to new insights about what energy is for. Yet we also hope that it has demonstrated the importance of considering how multiple projects (as well as multiple practices, technologies, institutions and groups of people) can be looked at together. Better understandings of social life are developed incrementally. Developing innovative approaches to how we might bring together understandings and knowledge is therefore an important part of the creativity of research. By addressing a broad range of cases, and drawing out some of the points of intersection between them, we hope to have provided fodder for imagination and encouraged further efforts to produce innovative future accounts of what energy is for and how this is changing.

\section{Bibliography}

Castree, N., and G. Watt. 2017. What kind of socio-technical research for what sort of influence on energy policy? Energy Research and Social Science 26: 87-90.

Cooper, A.C.G. 2017. Building physics into the social: Enhancing the policy impact of energy studies and energy social science research. Energy Research and Social Science 26: 80-86.

Flyvbjerg, B. 2001. Making social science matter: Why social inquiry fails and how it can succeed again. Cambridge: Cambridge University Press.

Mills, C.W. 1970. The sociological imagination. Harmondsworth: Penguin.

Reckwitz, A. 2002. Toward a theory of social practices: A development in culturalist theorizing. European Journal of Social Theory 5: 243-263.

Schatzki, T.R. 2010. The timespace of human activity: On performance, society, and history as indeterminate teleological events. Lanham: Lexington Books.

Shove, E., M. Pantzar, and M. Watson. 2012. The dynamics of social practice: Everyday life and how it changes. London: Sage.

Allison Hui is an Academic Fellow in Sociology at the DEMAND (Dynamics of Energy, Mobility and Demand) Research Centre at Lancaster University, UK. Her research examines transformations in everyday life in the context of changing global mobilities, focusing particularly on theorising social practices, consumption and travel. Recent co-edited publications include The Nexus of Practices (Routledge, 2017) and Traces of a Mobile Field (Routledge, 2017). 
Rosie Day is a Senior Lecturer in Human Geography at the University of Birmingham, UK. Her research interests centre around social inequalities in access to and experience of environmental and energy resources, with a related interest in environments of ageing and older age. She works collaboratively on a number of multidisciplinary projects in diverse international contexts.

Gordon Walker is Co-Director of the DEMAND (Dynamics of Energy, Mobility and Demand) Research Centre at Lancaster University and Professor in the Lancaster Environment Centre. He has wide-ranging expertise on the social and spatial dimensions of sustainable energy issues, sustainable social practices and cross cutting issues and theories of climate, energy and environmental justice.

Open Access This chapter is distributed under the terms of the Creative Commons Attribution 4.0 International License (http://creativecommons.org/ licenses/by/4.0/), which permits use, duplication, adaptation, distribution and reproduction in any medium or format, as long as you give appropriate credit to the original author(s) and the source, provide a link to the Creative Commons license and indicate if changes were made.

The images or other third party material in this chapter are included in the chapter's Creative Commons license, unless indicated otherwise in a credit line to the material. If material is not included in the chapter's Creative Commons license and your intended use is not permitted by statutory regulation or exceeds the permitted use, you will need to obtain permission directly from the copyright holder. 